\title{
BMJ Open Predictive value of echocardiographic left atrial size for incident stoke and stroke cause mortality: a population- based study
}

\author{
Tan Li, ${ }^{1}$ Guangxiao Li (1) , ${ }^{2}$ Xiaofan Guo, ${ }^{3}$ Zhao Li, ${ }^{3}$ Jun Yang, ${ }^{1}$ Yingxian Sun (1) ${ }^{3}$
}

To cite: Li T, Li G, Guo X, et al. Predictive value of echocardiographic left atrial size for incident stoke and stroke cause mortality: a populationbased study. BMJ Open 2021;11:e043595. doi:10.1136/ bmjopen-2020-043595

- Prepublication history and additional materials for this paper is available online. To view these files, please visit the journal online (http://dx.doi. org/10.1136/bmjopen-2020043595).

Received 10 August 2020 Revised 18 February 2021 Accepted 23 February 2021

A) Check for updates

C Author(s) (or their employer(s)) 2021. Re-use permitted under CC BY-NC. No commercial re-use. See rights and permissions. Published by BMJ.

${ }^{1}$ Department of Cardiovascular Ultrasound, The First Hospital of China Medical University,

Shenyang, China

${ }^{2}$ Department of Medical Record Management Center, The First Hospital of China Medical University, Shenyang, China ${ }^{3}$ Department of Cardiology, The First Hospital of China Medical University, Shenyang, China

Correspondence to Dr Yingxian Sun; yxsun@cmu.edu.cn

\section{ABSTRACT}

Objectives To investigate the associations between echocardiographic left atrial (LA) size and incident stoke and stroke cause mortality among a rural population in China.

Design A prospective study.

Setting and participants Based on the Northeast China Rural Cardiovascular Health Study, we selected a total of 10041 participants aged $\geq 35$ years who agreed to have transthoracic echocardiography at baseline and were successfully followed up for incident stoke and stroke cause mortality.

Primary outcome measure The outcomes were stroke and stroke cause death according to medical records and death certificates during the follow-up period.

Results LA enlargement (LAE) group had a higher prevalence of cardiovascular disease than normal LA diameter (LAD) group. After excluding individuals who had a prior stroke, subjects with LAE showed higher incident rates of stroke and its mortality in the overall and specific stratified analyses (all $p<0.05$ ). Kaplan-Meier analysis revealed that LAE could predict stroke incidence and stroke-free survival, but the association was no longer observed after the adjustment for potential confounding factors. Cox regression analysis reported that per $1 \mathrm{SD}$ increment in $\mathrm{LAD}$ and $\mathrm{LAD}$ / body surface area (BSA) was associated with an increased incidence of stroke (LAD: $H R=1.20,95 \% \mathrm{Cl} 1.08$ to 1.33 , $\mathrm{p}<0.001$; LAD/BSA: HR=1.22, 95\% Cl 1.11 to $1.35, \mathrm{p}<0.001)$ and stroke cause mortality (LAD: $H R=1.27,95 \% \mathrm{Cl} 1.08$ to $1.50, p<0.01$; LAD/BSA: $\mathrm{HR}=1.41,95 \% \mathrm{Cl} 1.20$ to 1.65 , $\mathrm{p}<0.001)$ in the total population, and similar trends were found in both genders (all $p<0.05$ ). LAD or LAD/BSA was related to ischaemic and haemorrhagic stroke incidence, and the risk of ischaemic and haemorrhagic stroke mortality (all $p<0.05)$. The dose-response curves further suggested linear associations between $L A D, L A D / B S A$ and the incidence of stroke and subsequent mortality in the general population (all $\mathrm{p}<0.05$ ).

Conclusions Our population-based study implied that LA size, especially LAD and LAD/BSA, might be useful echocardiographic biomarkers that had the potential to predict incident stroke and stroke cause mortality.

\section{INTRODUCTION}

Stroke is a global catastrophic condition with a great burden of disability and mortality. It has been predicted that there will be almost 12 million stroke deaths and 70 million stroke
Strengths and limitations of this study

- This was a large population-based prospective study, providing adequate data and sample size to delineate the study objective.

- The left atrial (LA) size determined by transthoracic echocardiography was treated as dichotomous and continuous variables, respectively.

- Multiple clinical covariates and echocardiographic parameters were adjusted in Cox proportional hazards models and multivariate logistic regression analyses.

- Restricted cubic spline functions were applied to draw the dose-response curves that indicated the associations between LA diameter (LAD), LAD/body surface area (BSA) and the risk of stroke or stroke cause mortality.

- This was a single centre study and further multicentre prospective ones with longer periods of follow-up should be conducted.

survivors by 2030. ${ }^{12}$ Pathologically, stroke can be classified into ischaemic stroke (around $80 \%$ ) and haemorrhage stroke (around $20 \%){ }^{3}$ To discern the high risks of stroke subjects and well manage stroke risk factors has a crucial individual and social impact. ${ }^{4}$

Left atrial (LA) dilation, the hallmark of LA remodelling, has been noted to be of clinical value for predicting the likelihood of cardiovascular events and all-cause mortality beyond potential risk factors. ${ }^{56}$ Among various quantification methods to assess LA size, the antero-posterior LA diameter (LAD) determined by transthoracic echocardiography is known to be the simplest and most reproducible measurement, and widely used in daily clinical practice and research. ${ }^{7}$ As a risk factor for ischaemic stroke, LA enlargement (LAE) has been discussed in many studies, but the data from large-scale prospective cohorts are limited and conclusions vary. Several longitudinal studies identified that LAD was associated with incident stroke, ${ }^{489}$ but other 
cohort studies found no relationship. ${ }^{10-13}$ Some authors also investigated whether this association was influenced by gender. Benjamin et al and Di Tullio et al revealed an independently predictive effect of LAE on ischaemic stroke risk only in men, ${ }^{14}{ }^{15}$ whereas others found that LAE predicted stroke only in women. ${ }^{11}{ }^{16}$ However, above researches just concentrated on non-Asian populations, and there were a lack of data regarding the importance of LA size in predicting incident stoke in Asians. Additionally, a link of LA size to stroke cause mortality and haemorrhagic stroke incidence has not been sufficiently described in the literature. Up to now, only one Asian community cohort from Japan demonstrated that LAE was an independent predictor of stroke/systemic embolism in patients with atrial fibrillation (AF). ${ }^{17}$ Considering the differences in epidemiological characteristics, the mechanisms of strokes in China can also vary from other countries. Therefore, it is the necessity to discern the association between LA size and incident stroke and its mortality in a Chinese population.

In the current study, based on the Northeast China Rural Cardiovascular Health Study (NCRCHS) with a median followed up of 4.66 years, we aimed to determine the role of LA size, regarded as dichotomous and continuous variables respectively, on the prediction of incident stroke and stroke cause mortality in the total and stratified analyses. Then, we sought to find out whether there were some continuous dose-response associations between LAD, LAD/body surface area (BSA) and the risk of stroke and its mortality in the general population. Our data may serve to enhance the risk stratification for stroke, and help guide targeting of primary prevention efforts.

\section{MATERIALS AND METHODS \\ Study population}

NCRCHS is a community-based prospective cohort study carried out in rural areas of Northeast China. The design and inclusion criteria of the study have been described previously. ${ }^{18} 19$ In brief, a total of 11956 participants aged $\geq 35$ years were recruited from Dawa, Zhangwu and Liaoyang counties in Liaoning province between 2012 and 2013, using a multi-stage, randomly stratified and cluster-sampling scheme. We excluded 341 subjects who refused to have an echocardiography performed at baseline. In 2015 and 2017, participants were invited to attend a follow-up study. Of the 11615 subjects, 10377 participants consented and were eligible for our follow-up study. A total of 10041 participants (96.8\%) completed at least one follow-up visit and were available for our analysis. Detailed information was collected at baseline for each participant.

\section{Data collection}

At baseline, detailed information on demographic characteristics, lifestyle factors and medical history were acquired by interview with a standardised questionnaire. Smoking was defined as having smoked at least one cigarette per day for more than 6 months. Drinking was defined as having alcohol consumption at least two times a week for more than 1 year. Weight and height were measured with participants in light weight clothing and without shoes. BSA was calculated as $(0.0061 \times$ height $(\mathrm{cm})+0.0128 \times$ wt (kg)-0.1529). Blood pressure was assessed three times with participants seated after at least $5 \mathrm{~min}$ of rest using a standardised automatic electronic sphygmomanometer (HEM-907; Omron, Tokyo, Japan). Hypertension was defined as systolic blood pressure $\geq 140 \mathrm{~mm} \mathrm{Hg}$ and/or diastolic blood pressure $\geq 90 \mathrm{~mm} \mathrm{Hg}$, and/or use of antihypertensive medications. ${ }^{20}$ Fasting blood samples were collected in the morning from participants who had fasted at least 12 hours. Total cholesterol (TC), triglyceride (TG), low-density lipoprotein cholesterol (LDL-C), highdensity lipoprotein cholesterol (HDL-C), fasting plasma glucose (FPG), serum creatinine and other routine blood biochemical indexes were analysed enzymatically. Diabetes was defined as FPG $\geq 7 \mathrm{mmol} / \mathrm{L}(126 \mathrm{mg} / \mathrm{dL})$ and/or being on medication for diabetes. ${ }^{21}$ Dyslipidaemia was defined as serum $\mathrm{TC} \geq 6.21 \mathrm{mmol} / \mathrm{L}(240 \mathrm{mg} / \mathrm{dL})$, or $\mathrm{TG} \geq 2.26 \mathrm{mmol} / \mathrm{L}(200 \mathrm{mg} / \mathrm{dL})$, or LDL-C $\geq 4.16 \mathrm{mmol} / \mathrm{L}$ $(160 \mathrm{mg} / \mathrm{dL})$, or HDL-C $<1.03 \mathrm{mmol} / \mathrm{L}(40 \mathrm{mg} / \mathrm{dL})$ and/ or under taking hypolipidaemic drugs. ${ }^{22}$

Transthoracic echocardiographic examination was performed using a commercially available Doppler echocardiograph (Vivid, GE Healthcare, USA) with a 3.0 $\mathrm{MHz}$ transducer, including M-mode, two-dimensional, spectral and colour Doppler. The echo did not have a clinical indication, but rather was done as part of a longitudinal cohort study at specific study visits. Echocardiographic analyses and readings were conducted by three doctors specialised in echocardiography, and there was a high degree of intra-observer and inter-observer reproducibility for interpretation of the echoes. Under the guideline of the American Society of Echocardiography, ${ }^{7}$ the parasternal long-axis view was measured to record antero-posterior LAD, interventricular septal thickness, left ventricular (LV) end-diastolic internal dimension (LVIDd), LV end-systolic internal dimension (LVIDs) and posterior wall thickness (PWTd). LV mass (LVM) was calculated by the formula: LVM $=0.8 \times[1.04$ (IVST$\mathrm{d}+$ PWTd+LVIDd $)^{3}-$ LVIDd $\left.\left.^{3}\right\}\right]+0.6 \mathrm{~g}$. LVM was divided by BSA to acquire LVM index (LVMI). Normally, the LV end-diastolic volume (LVEDV) and LV end-systolic volume (LVESV) were estimated by Teichholz equations: LVEDV $(\mathrm{mL})=\operatorname{LVIDd}^{3} \times 7.0 /(2.4+\mathrm{LVIDd}), \mathrm{LVESV}$ $(\mathrm{mL})=$ LVIDs $^{3} \times 7.0 /(2.4+\mathrm{LVIDs})$. When there were abnormalities in cardiac structure and function, we used the biplane Simpson's rule for volume calculations from both the apical four-chamber and two-chamber views. LV ejection fraction (LVEF) was calculated as [(LVEDV-LVESV) / LVEDV] $\times 100 \%$. We applied pulsed-wave Doppler to record the early diastolic peak flow $(\mathrm{E})$ of mitral valve in the apical four-chamber view. Pulsed-wave tissue Doppler imaging was used to assess the peak early ( $\left.\mathrm{e}^{\prime}\right)$ diastolic velocities of the septal and lateral mitral annulus in the 
apical four-chamber view. Subsequently, the average from the septal and lateral velocities was used to obtain the $\mathrm{E} / \mathrm{e}^{\prime}$. LAE was defined as $\mathrm{LAD}>39 \mathrm{~mm}$ and $>37 \mathrm{~mm}$ for men and women, respectively. ${ }^{23}$

\section{Judgment and definition of clinical outcomes}

The median follow-up time was 4.66 (4.36-4.93) years. The number of effective follow-up cases was 10041, and follow-up rate was $96.8 \%$. In the present study, an incident event was defined as a new onset of stroke or stroke cause death during follow-up period. The subjects with history of stroke at baseline were excluded when we defined incident stroke and performed the survival analysis for the subsequent outcomes. Stroke was defined according to the WHO Multinational Monitoring of Trends and Determinants in Cardiovascular Disease criteria, ${ }^{24}$ as rapidly developing signs of focal or global disturbance of cerebral function, lasting more than 24 hours (unless interrupted by surgery or death) with no apparent nonvascular causes. Haemorrhagic stroke was defined as stroke cases with diagnosis of subarachnoid haemorrhage or intracerebral haemorrhage and ischaemic stroke was defined as stroke cases with diagnosis of thrombosis or embolism. Transient ischaemic attack and chronic cerebral vascular disease were excluded. For all participants reporting possible diagnoses or death, all available clinical information was collected including medical records and death certificates. All materials were independently reviewed and adjudicated by the end-point assessment committee.

\section{Statistical analysis}

All statistical analyses were performed with SPSS V.23.0 software. Continuous variables were reported as mean values $\pm \mathrm{SD}$, and categorical variables were represented as numbers and percentages. Differences among categories were evaluated using the t-test, non-parameter test, $\chi^{2}$-test or Fisher's exact test as appropriate. Kaplan-Meier estimates were adopted to calculate the cumulative incidence of stroke and stroke-free survival for each group, and log-rank test was used to compare the differences in estimates. Cox proportional hazards models were used to identify the associations of LAE, LAD and LAD/BSA with the incidence of stroke and stroke cause mortality with HRs and 95\% CIs calculated after adjusting the potential confounders. As restricted cubic spline makes it possible to characterise the flexible and visible dose-response relationship between an exposure and outcome, ${ }^{25}$ this method was used to depict the dose-response associations between LAD, LAD/BSA and the risk of stroke or stroke cause mortality based on logistic regression models. The adjusted covariates included age, gender and other potential confounders. All tests were two-tailed and $p<0.05$ indicated statistical significance.

\section{Patient and public involvement}

No patients were involved in setting the research questions or outcome measures, and they were not involved in the design or performance of this study. No plans were set to disseminate the research results to study participants.

\section{RESULTS}

\section{Baseline clinical and echocardiographic characteristics}

In this study, there were 8915 subjects with normal LAD and 1126 ones with LAE. Except for LVEF and statin use, there were statistical differences in the distribution of baseline clinical and echocardiographic parameters between two groups (all $\mathrm{p}<0.05)$, as shown in table 1 . Participants with LAE had a significantly increased prevalence of hypertension and AF compared with normal LAD group as would be anticipated.

\section{Incidences of clinical outcomes}

During a median follow-up of 4.66 years, 336 participants $(3.5 \%)$ developed new onset stroke and 106 subjects $(1.1 \%)$ died from stroke in the total cohort. The incident rates of overall stroke and mortality were statistically higher in participants with LAE than those in normal LAD group (all $\mathrm{p}<0.05$ ) (table 2 ). The total population was further subdivided according to gender and stroke type. Compared with normal LAD group, incidence of stroke in either male or female subjects with LAE was significantly higher, but only male subjects with LAE had higher incidence of stroke cause mortality (all $\mathrm{p}<0.05)$. Incident ischaemic stroke as well as haemorrhagic stroke cause mortality was significantly more frequent in participants with LAE (all p<0.05) (table 2). Additionally, stratified analyses by AF showed that only three subjects had incident stroke and stroke cause death in AF participants respectively, and LAE group displayed a significantly increased stroke incidence when compared with normal $\mathrm{LAD}$ group in the population without $\mathrm{AF}(\mathrm{p}<0.01)$ (online supplemental table 1).

\section{LA size for predicting stroke and stroke cause mortality}

Kaplan-Meier survival estimates revealed that participants with LAE had higher cumulative stroke incidence and worse stroke-free survival than those with normal LAD in overall population (figure 1). There was an association between LAE and stroke incidence in men or women, and a similar trend was observed for ischaemic stroke incidence (all $\mathrm{p}<0.01$ ) (figure 2). As described, LAE was significantly associated with worse stroke-free survival in men and ischaemic subtype during follow-up (all $\mathrm{p}<0.05$ ) (figure 3).

However, in the multivariable model after adjusting the confounding factors, the association between LAE and the risk of stroke or stroke cause mortality was no longer significant, as presented in table 3 and online supplemental table 2. When $\mathrm{LAD}$ and $\mathrm{LAD} / \mathrm{BSA}$ were treated as continuous variables, per $1 \mathrm{SD}$ increase in $\mathrm{LAD}$ or $\mathrm{LAD} / \mathrm{BSA}$ was significantly associated with an elevated risk of stroke (LAD: HR=1.20, 95\% CI 1.08 to 1.33 , $\mathrm{p}<0.001 ; \mathrm{LAD} / \mathrm{BSA}$ : $\mathrm{HR}=1.22,95 \%$ CI 1.11 to $1.35, \mathrm{p}<0.001)$ and stroke cause mortality (LAD: HR=1.27, 95\% CI 1.08 to $1.50, \mathrm{p}<0.01$; 
Table 1 Baseline clinical and echocardiographic characteristics of study participants stratified by LAE status

\begin{tabular}{|c|c|c|c|}
\hline & Normal LAD ( $\mathrm{n}=8915)$ & LAE $(n=1126)$ & $P$ value \\
\hline Age, years & $53.38 \pm 10.39$ & $57.45 \pm 10.45$ & $<0.001$ \\
\hline Male, n (\%) & $4229(47.4)$ & $414(36.8)$ & $<0.001$ \\
\hline $\mathrm{BSA}, \mathrm{m}^{2}$ & $1.64 \pm 0.17$ & $1.72 \pm 0.19$ & $<0.001$ \\
\hline Smoking, n (\%) & 3246 (36.4) & $304(27.0)$ & $<0.001$ \\
\hline Drinking, n (\%) & $2082(23.4)$ & $194(17.2)$ & $<0.001$ \\
\hline Heart rate, bmp & $78.44 \pm 13.31$ & $77.35 \pm 13.49$ & 0.01 \\
\hline SBP, mm Hg & $140.15 \pm 22.47$ & $156.53 \pm 26.51$ & $<0.001$ \\
\hline DBP, mm Hg & $81.55 \pm 11.52$ & $86.31 \pm 13.32$ & $<0.001$ \\
\hline Hypertension, n (\%) & $4174(46.8)$ & $853(75.8)$ & $<0.001$ \\
\hline Medication for hypertension, $\mathrm{n}(\%)$ & $1134(12.7)$ & $351(31.2)$ & $<0.001$ \\
\hline History of CHD, n (\%) & $398(4.5)$ & $115(10.2)$ & $<0.001$ \\
\hline History of AF, n (\%) & $55(0.6)$ & $32(2.8)$ & $<0.001$ \\
\hline History of HF, n (\%) & $61(0.7)$ & $35(3.1)$ & $<0.001$ \\
\hline History of stroke, n (\%) & 298 (3.3) & $78(6.9)$ & $<0.001$ \\
\hline History of heart valve diseases, $\mathrm{n}(\%)$ & $434(4.9)$ & $153(13.6)$ & $<0.001$ \\
\hline $\mathrm{TC}, \mathrm{mmol} / \mathrm{L}$ & $5.23 \pm 1.09$ & $5.38 \pm 1.14$ & $<0.001$ \\
\hline $\mathrm{TG}, \mathrm{mmol} / \mathrm{L}$ & $1.57 \pm 1.45$ & $1.81 \pm 1.43$ & $<0.001$ \\
\hline LDL-C, mmol/L & $2.94 \pm 0.83$ & $3.09 \pm 0.87$ & $<0.001$ \\
\hline HDL-C, mmol/L & $1.43 \pm 0.40$ & $1.35 \pm 0.35$ & $<0.001$ \\
\hline Dyslipidaemia, n (\%) & 3230 (36.2) & $483(42.9)$ & $<0.001$ \\
\hline eGFR (mL/min.1.73 m²) & $93.94 \pm 15.31$ & $89.20 \pm 16.19$ & $<0.001$ \\
\hline FPG, mmol/L & $5.85 \pm 1.54$ & $6.22 \pm 2.06$ & $<0.001$ \\
\hline Diabetes, n (\%) & $859(9.6)$ & $197(17.5)$ & $<0.001$ \\
\hline Medication for diabetes, n (\%) & $300(3.4)$ & $73(6.5)$ & $<0.001$ \\
\hline $\mathrm{LAD}, \mathrm{cm}$ & $3.29 \pm 0.31$ & $4.08 \pm 0.34$ & $<0.001$ \\
\hline LAD/BSA, $\mathrm{cm} / \mathrm{m}^{2}$ & $2.02 \pm 0.22$ & $2.40 \pm 0.31$ & $<0.001$ \\
\hline LVMI, $g / \mathrm{m}^{2}$ & $81.77 \pm 16.32$ & $100.20 \pm 27.71$ & $<0.001$ \\
\hline LVEF, \% & $61.80 \pm 10.10$ & $61.39 \pm 11.18$ & 0.24 \\
\hline$E / e^{\prime}$ & $9.06 \pm 3.98$ & $10.73 \pm 5.61$ & $<0.001$ \\
\hline Aspirin use, $\mathrm{n}(\%)$ & $75(0.8)$ & $18(1.6)$ & 0.01 \\
\hline Statin use, $\mathrm{n}(\%)$ & $23(0.3)$ & $4(0.4)$ & 0.54 \\
\hline
\end{tabular}

$\mathrm{AF}$, atrial fibrillation; BSA, body surface area; CHD, coronary heart disease; DBP, diastolic blood pressure; eGFR, estimated glomerular filtration rate ; FPG, fasting plasma glucose; HDL-C, high-density lipoprotein cholesterol; LAD, left atrial diameter; LAE, left atrial enlargement; LDL-C, low-density lipoprotein cholesterol; LVEF, left ventricular ejection fraction; LVMI, left ventricular mass index; SBP, systolic blood pressure; TC, total cholesterol; TG, triglyceride.

LAD/BSA: HR=1.41, 95\% CI 1.20 to $1.65, \mathrm{p}<0.001)$ in overall population (table 3). Further stratified analyses showed that $\mathrm{LAD}$ or LAD/BSA was closely related to the incidence of stroke and stroke cause mortality in both male and female subjects (all $\mathrm{p}<0.05)$ (table 3 ). After the adjustment for the potential confounders, a relationship of LAD with incident ischaemic stroke, and a significant association between LAD/BSA and the risk of both ischaemic stroke and its cause death were observed, while $\mathrm{LAD}$ and LAD/BSA were correlated with an increased risk of haemorrhagic stroke and its mortality (all $\mathrm{p}<0.05$ ) (table 3). When the participants with AF were excluded, the predictive value of $\mathrm{LAD}$ and $\mathrm{LAD} / \mathrm{BSA}$ for incident stroke and stroke cause mortality remained significant in the subjects without AF (all $\mathrm{p}<0.01$ ) (online supplemental table 2).

Dose-response analyses of the associations between LAD, LAD/BSA and the risk of stroke or stroke cause mortality

After the adjustment for age, gender, BSA, smoking, drinking, heart rate, history of coronary heart disease (CHD), history of AF, history of heart failure (HF), history of heart valve diseases, hypertension, diabetes, medication for hypertension and diabetes, dyslipidaemia, 
Table 2 Incidence of stroke and stroke cause mortality in study participants

\begin{tabular}{|c|c|c|c|c|}
\hline \multirow[b]{2}{*}{ Outcomes } & \multirow[b]{2}{*}{ Overall $(n=9665)$} & \multicolumn{2}{|l|}{ Incidence } & \multirow[b]{2}{*}{$\mathbf{P}$ value } \\
\hline & & Normal LAD $(n=8617)$ & LAE $(n=1048)$ & \\
\hline Stroke, $\mathrm{n}(\%)^{*}$ & $336(3.5)$ & $281(3.3)$ & $55(5.3)$ & $<0.01$ \\
\hline Female, n (\%) & $134(1.4)$ & $105(1.2)$ & $29(2.8)$ & $<0.01$ \\
\hline Ischaemic stroke, n (\%) & $261(2.7)$ & $218(2.5)$ & $43(4.1)$ & $<0.01$ \\
\hline Stroke cause mortality, $\mathrm{n}(\%)^{\star}$ & $106(1.1)$ & $86(1.0)$ & $20(1.9)$ & $<0.01$ \\
\hline Male, n (\%) & $63(0.7)$ & $52(0.6)$ & $11(1.0)$ & 0.01 \\
\hline Female, n (\%) & $43(0.4)$ & $34(0.4)$ & $9(0.9)$ & 0.11 \\
\hline Ischaemic stroke, n (\%) & $46(0.5)$ & $38(0.4)$ & $8(0.8)$ & 0.15 \\
\hline Haemorrhagic stroke, n (\%) & $50(0.5)$ & $40(0.5)$ & $10(1.0)$ & 0.04 \\
\hline
\end{tabular}

Subjects with history of stroke at baseline were excluded.

*10 patients with unknown stroke type.

LAD, left atrial diameter; LAE, left atrial enlargement.

estimated glomerular filtration rate (eGFR), statin use, aspirin use, LVMI, LVEF, E/e' as appropriate, the doseresponse curves indicated linear associations between $\mathrm{LAD}, \mathrm{LAD} / \mathrm{BSA}$ and stroke risk in the general population (for LAD, test for overall association: $p<0.001$; test

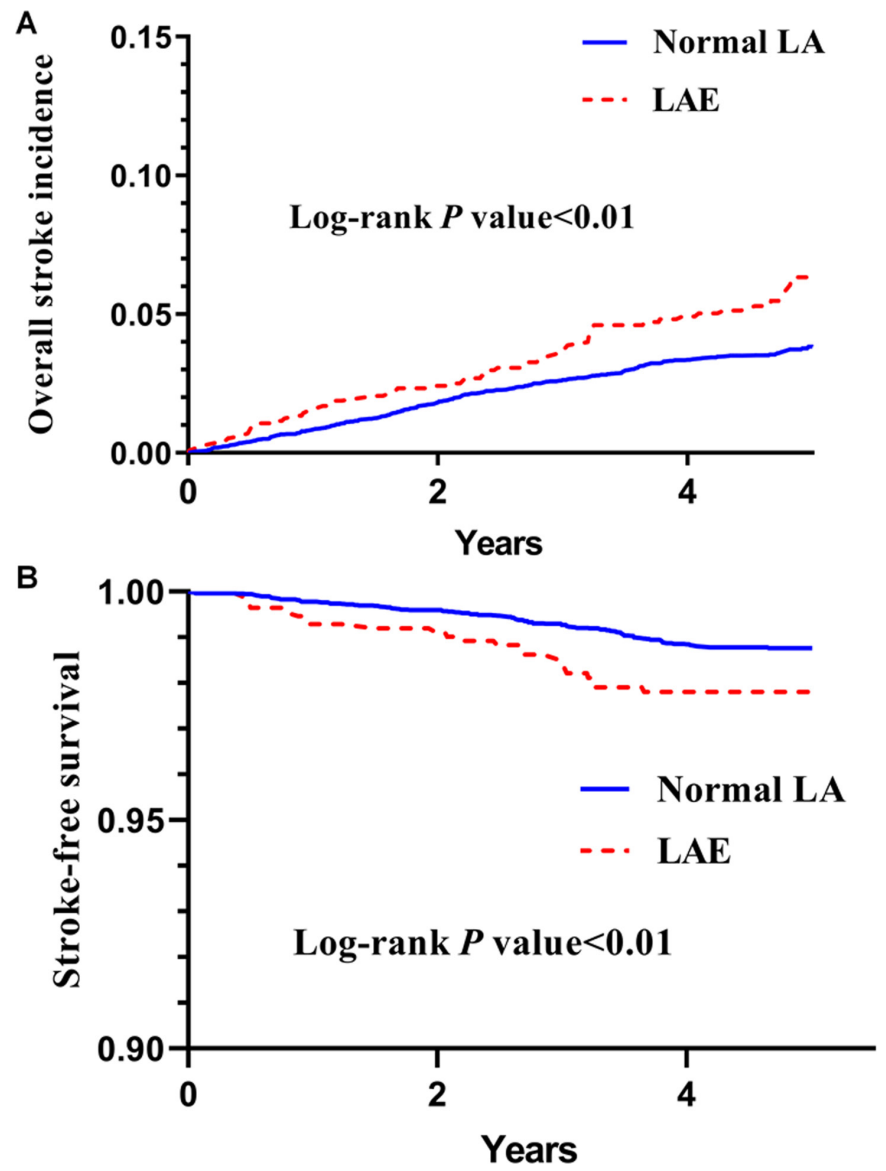

Figure 1 Kaplan-Meier survival curves for stroke incidence (A) and stroke-free survival $(B)$ in overall population according to LA size. LA, left atrial; LAE, LA enlargement. for non-linear association: $\mathrm{p}=0.80$, Akaike information criterion $(\mathrm{AIC})=5984.42$; for $\mathrm{LAD} / \mathrm{BSA}$, test for overall association: $\mathrm{p}<0.001$; test for non-linear association: $\mathrm{p}=0.72$, $\mathrm{AIC}=5984.42$ ) (figures 4 and 5) (online supplemental tables 3 and 4). Similarly, significant linear doseresponse associations between $\mathrm{LAD}, \mathrm{LAD} / \mathrm{BSA}$ and the risk of stroke cause death were observed (for LAD, test for overall association: $\mathrm{p}<0.001$; test for non-linear association: $\mathrm{p}=0.12, \mathrm{AIC}=1927.08$; for $\mathrm{LAD} / \mathrm{BSA}$, test for overall association: $\mathrm{p}<0.001$; test for non-linear association: $\mathrm{p}=0.28, \mathrm{AIC}=1927.08$ ) (figures 4 and 5) (online supplemental tables 3 and 4 ).

\section{DISCUSSION}

In this large Chinese population-based cohort, we showed a significantly higher incidence of stroke and stroke cause mortality in LAE group compared with normal LAD group in the overall and stratified analyses. Interestingly, our study further demonstrated that LAD and LAD/ BSA rather than LAE were possible predictors of incident stroke and its mortality in the general population and specific subgroups, and there could be linear doseresponse associations between $\mathrm{LAD}, \mathrm{LAD} / \mathrm{BSA}$ and the risk of stroke and stroke cause mortality.

Enlarged LA size appears to be increasingly regarded as a prognostic biomarker for thromboembolic and cardiovascular events. $^{26} 27$ A number of populationbased studies tried to evaluate the role of LA size in predicting stroke, obtaining controversial results. Some studies supported the association between LA size and stroke risk, ${ }^{489}$ whereas others did not. ${ }^{10-13}$ Froehlich et al conducted a meta-analysis to reveal that indexed LAD could be an important predictor of stroke without adjustment for cardiovascular risk factors. ${ }^{26} \mathrm{~A}$ recent systematic review involving six cohorts concluded that LAE was related to an increased and graded risk of stroke 

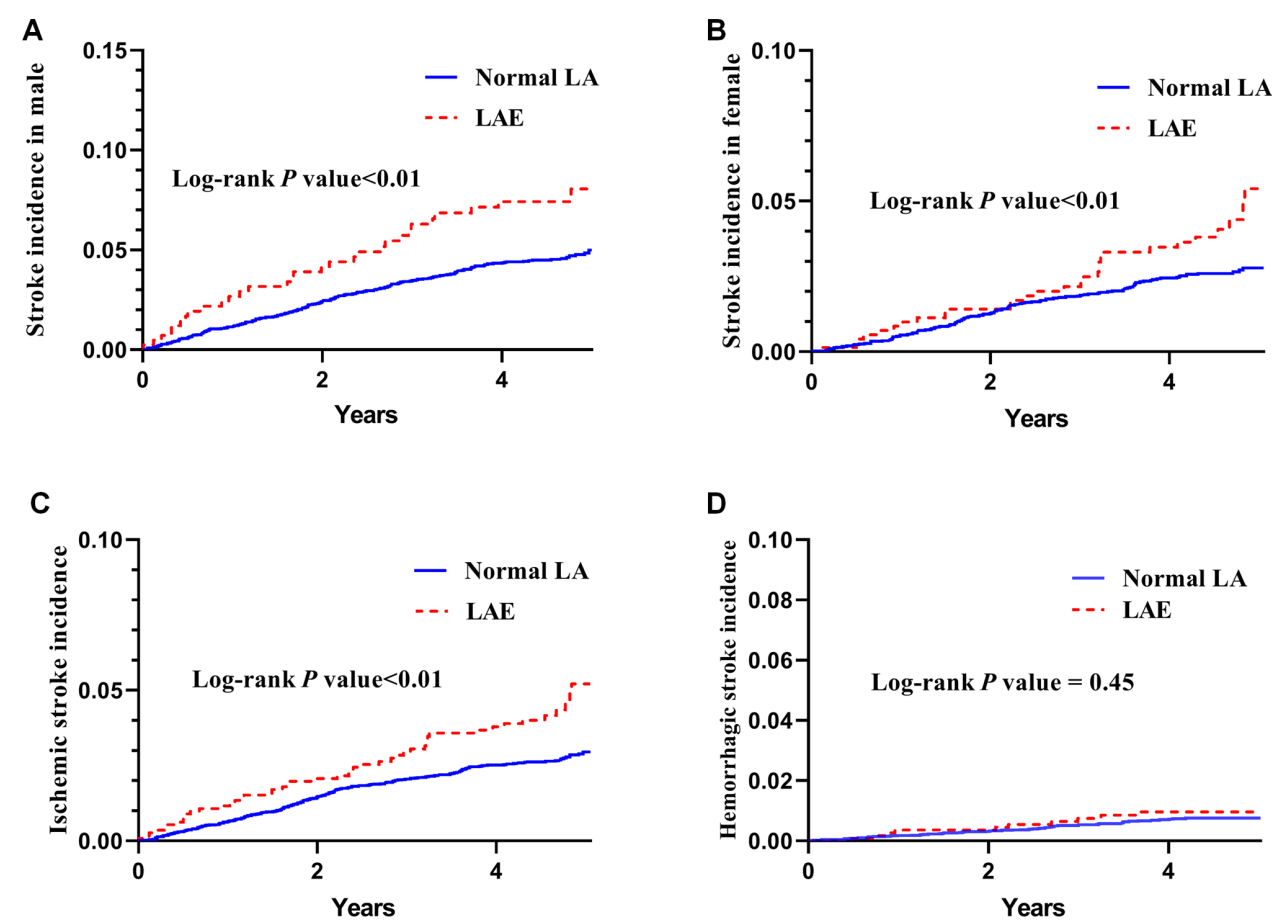

Figure 2 Kaplan-Meier survival curves for stroke incidence in men (A) and women (B), and ischaemic (C) and haemorrhagic (D) stroke incidence according to LA size. LA, left atrial; LAE, LA enlargement.

independent of age, sex, hypertension and diabetes. ${ }^{2}$ In our study, when LAD was dichotomised based on sexspecific partition values, subjects with LAE exhibited elevated incident rates of stroke and its mortality in the overall and stratified comparisons. The Kaplan-Meier curves revealed that LAE was significantly associated with higher stroke incidence in male, female and ischaemic subtype, as well as worse stroke-free survival in male and ischaemic stroke. Of note, our multivariate analysis did not confirm LAE as an independent predictor for stroke and long-term survival. The reasons for these discordant results might be the ethnicity-related differences, study design heterogeneity, disparities in measurements, indexations and cut-off points to define LAE, and diverse adjustment factors. Another possibility is that the link between LAE and the risk of stroke and its cause death
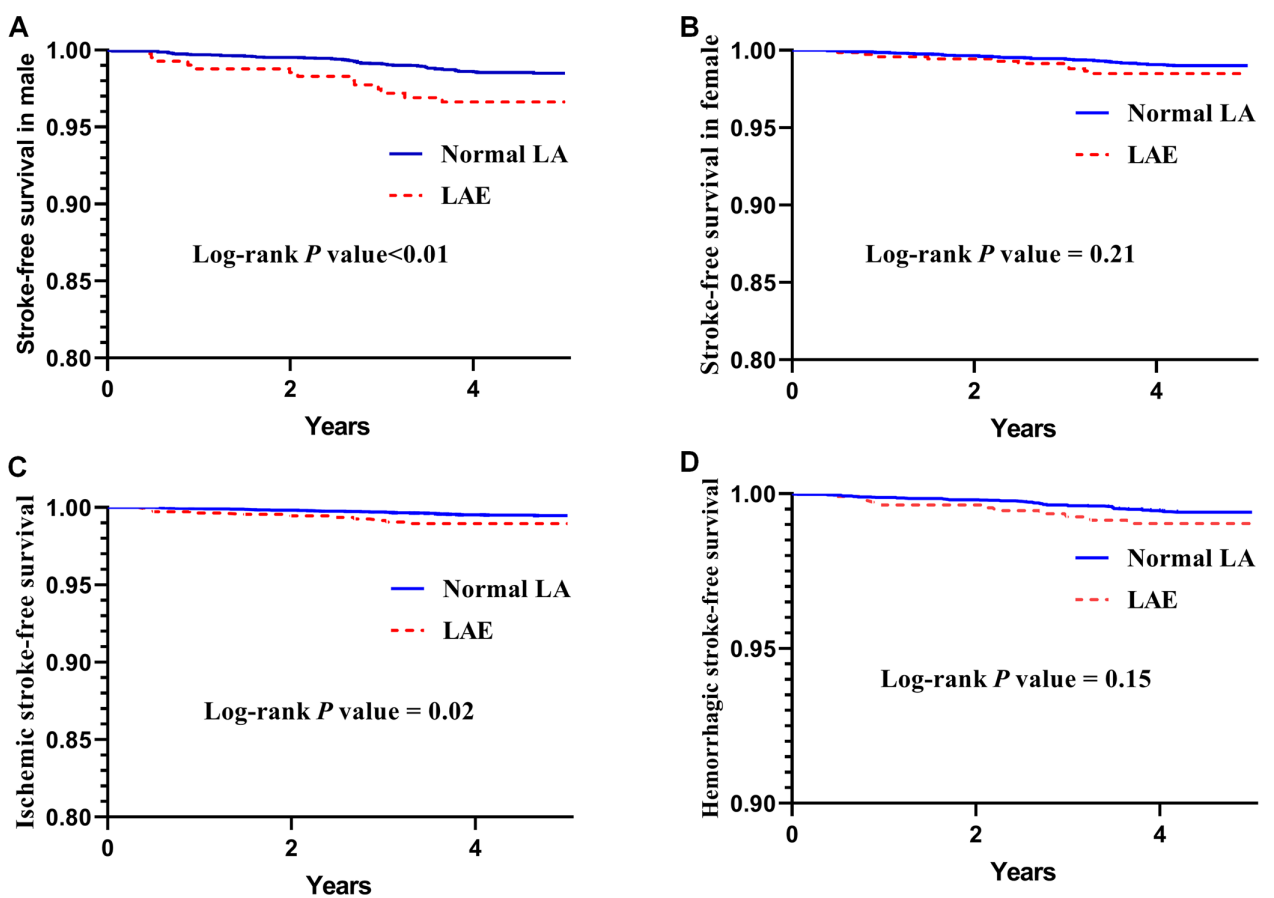

Figure 3 Kaplan-Meier survival curves for stroke-free survival in men $(A)$ and women $(B)$, and ischaemic $(C)$ and haemorrhagic (D) stroke-free survival according to LA size. LA, left atrial; LAE, LA enlargement. 
Table 3 LA size for the prediction of stroke and stroke cause mortality during follow-up

\begin{tabular}{|c|c|c|c|c|c|c|}
\hline \multirow[b]{2}{*}{ Subgroups } & \multicolumn{3}{|l|}{ Stroke* } & \multicolumn{3}{|c|}{ Stroke cause mortality* } \\
\hline & $\begin{array}{l}\text { Cases/non- } \\
\text { cases }\end{array}$ & $\begin{array}{l}\text { Incidence HR } \\
\text { (95\% Cl) }\end{array}$ & $P$ value & $\begin{array}{l}\text { Cases/non- } \\
\text { cases }\end{array}$ & $\begin{array}{l}\text { Incidence HR } \\
(95 \% \mathrm{Cl})\end{array}$ & $P$ value \\
\hline \multicolumn{7}{|l|}{ Overall } \\
\hline Normal LAD & $281 / 8336$ & Reference & - & $86 / 8531$ & Reference & - \\
\hline LAE & $55 / 993$ & 1.17 (0.86 to 1.58$)$ & 0.31 & $20 / 1028$ & 1.37 (0.82 to 2.28$)$ & 0.22 \\
\hline LAD, per 1 SD increase & - & 1.20 (1.08 to 1.33$)$ & $<0.001$ & - & 1.27 (1.08 to 1.50$)$ & $<0.01$ \\
\hline LAD/BSA, per 1 SD increase & - & 1.22 (1.11 to 1.35$)$ & $<0.001$ & - & 1.41 (1.20 to 1.65$)$ & $<0.001$ \\
\hline \multicolumn{7}{|l|}{ Gender } \\
\hline \multicolumn{7}{|l|}{ Male } \\
\hline Normal LAD & $176 / 3904$ & Reference & - & $52 / 4028$ & Reference & - \\
\hline LAE & $26 / 359$ & 1.05 (0.68 to 1.61$)$ & 0.84 & $11 / 374$ & 1.41 (0.71 to 2.79$)$ & 0.33 \\
\hline LAD, per $1 S D$ increase & - & 1.14 (1.00 to 1.29$)$ & 0.05 & - & 1.21 (0.99 to 1.48$)$ & 0.07 \\
\hline LAD/BSA, per $1 \mathrm{SD}$ increase & - & 1.19 (1.04 to 1.36$)$ & 0.01 & - & 1.35 (1.10 to 1.65$)$ & $<0.01$ \\
\hline \multicolumn{7}{|l|}{ Female } \\
\hline Normal LAD & $105 / 4432$ & Reference & - & $34 / 4503$ & Reference & - \\
\hline LAE & 29/634 & $1.28(0.83$ to 1.97$)$ & 0.27 & $9 / 654$ & 1.32 (0.61 to 2.86$)$ & 0.48 \\
\hline LAD, per $1 S D$ increase & - & 1.29 (1.08 to 1.53$)$ & $<0.01$ & - & 1.38 (1.03 to 1.84$)$ & 0.03 \\
\hline LAD/BSA, per 1 SD increase & - & 1.24 (1.05 to 1.46$)$ & 0.01 & - & 1.45 (1.11 to 1.91$)$ & $<0.01$ \\
\hline \multicolumn{7}{|l|}{ Stroke type } \\
\hline \multicolumn{7}{|l|}{ Ischaemic stroke } \\
\hline Normal LAD & 218/8391 & Reference & - & $38 / 8571$ & Reference & - \\
\hline $\mathrm{LAE}$ & 43/1003 & $1.15(0.82$ to 1.61$)$ & 0.42 & 8/1038 & 1.21 (0.55 to 2.67$)$ & 0.64 \\
\hline LAD, per $1 \mathrm{SD}$ increase & - & 1.22 (1.09 to 1.38$)$ & $<0.01$ & - & 1.28 (0.99 to 1.64$)$ & 0.05 \\
\hline LAD/BSA, per 1 SD increase & - & 1.18 (1.05 to 1.34$)$ & $<0.01$ & - & 1.34 (1.06 to 1.71$)$ & 0.02 \\
\hline \multicolumn{7}{|l|}{ Haemorrhagic stroke } \\
\hline Normal LAD & $55 / 8554$ & Reference & - & $40 / 8569$ & Reference & - \\
\hline LAE & 10/1036 & 1.03 (0.52 to 2.04$)$ & 0.94 & $10 / 1036$ & 1.38 (0.67 to 2.86$)$ & 0.39 \\
\hline LAD, per $1 S D$ increase & - & 1.27 (1.02 to 1.60$)$ & 0.04 & - & 1.35 (1.20 to 1.51$)$ & 0.01 \\
\hline LAD/BSA, per 1 SD increase & - & 1.29 (1.04 to 1.59$)$ & 0.02 & - & $1.38(1.10$ to 1.74$)$ & $<0.01$ \\
\hline
\end{tabular}

Subjects with history of stroke at baseline were excluded.

Adjusted for age, gender, BSA, smoking, drinking, heart rate, history of CHD, history of AF, history of HF, history of heart valve diseases, hypertension, diabetes, medication for hypertension and diabetes, dyslipidaemia, eGFR, statin use, aspirin use, leftventricular mass index, leftventricular ejection fraction, E/e' as appropriate.

*10 patients with unknown stroke type.

$A F$, atrial fibrillation; BSA, body surface area; CHD, coronary heart disease; eGFR, estimated glomerular filtration rate ; HF, heart failure; LAD, left atrial diameter; LAE, left atrial enlargement.

is mediated by a combination of other clinical covariates. ${ }^{28} 29$

When LA size was considered continuously, Cox regression analysis indicated that per $1 \mathrm{SD}$ increase in LAD or LAD/BSA was significantly correlated with an increased risk of stroke and stroke cause mortality in overall population after adjusting the potential confounders. The associations between LAD, LAD/BSA and the risk of stroke and its mortality were further verified by the dose-response analyses. Importantly, using the drawn dose-response curves, we could calculate the risks of developing stroke and subsequent mortality at any specific LAD or LAD/
BSA values, which would facilitate risk assessment and individualised management strategies. ${ }^{30}$ Above findings implied that close monitoring of echocardiographic LAD increment might add predictive information in the evaluation of stroke and its mortality, which needed to be validated through replication in other populations. However, Mosquera et al found that LAD/BSA raised the risk of stroke in $50.7 \%$ per $1 \mathrm{~cm} / \mathrm{m}^{2}$ increment in bivariate analysis, which association disappeared after multivariate regression. $^{31}$

Sexual differences in the association between LA size and stoke risk are of huge interest in current research. ${ }^{32} 33$ 

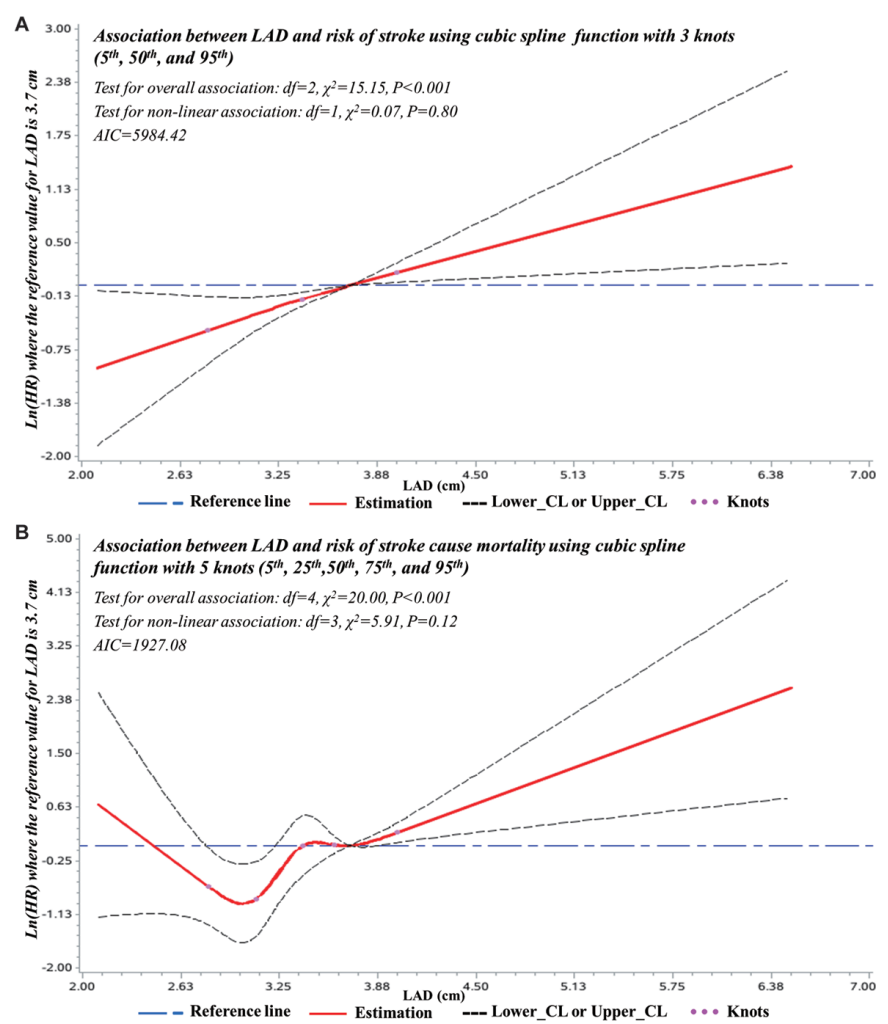

Figure 4 Adjusted dose-response associations between $L A D$ and the risk of stroke $(A)$ and stroke cause mortality (B). Adjusted for age, gender, BSA, smoking, drinking, heart rate, history of $\mathrm{CHD}$, history of $\mathrm{AF}$, history of $\mathrm{HF}$, history of heart valve diseases, hypertension, diabetes, medication for hypertension and diabetes, dyslipidaemia, eGFR, statin use, aspirin use, LVMI, LVEF, E/e' as appropriate. The Yaxis indicates the $\ln (\mathrm{HR})$ of stroke for any value of $L A D$ compared with the reference values. Dashed lines refer to the 95\% Cls. AIC, Akaike information criterion; CL, confidence interval; LAD, left atrial diameter; BSA, body surface area; $\mathrm{CHD}$, coronary heart disease; $\mathrm{AF}$, atrial fibrillation; $\mathrm{HF}$, heart failure; eGFR, estimated glomerular filtration rate; LVMI, left ventricular mass index; LVEF, left ventricular ejection fraction.

Some studies suggested that LAE could be considered as a potential risk factor for ischaemic stroke only in men, but others reported this association only in women. ${ }^{14}$ In our research, there were no obvious sex differences regarding the correlation analysis between $\mathrm{LAD}, \mathrm{LAD} / \mathrm{BSA}$ and incident stroke and stroke cause mortality. Furthermore, we found that $\mathrm{LAD}$ and $\mathrm{LAD} / \mathrm{BSA}$ could increase the risk of incident ischaemic stroke or its mortality. Interestingly, the present study also provided new evidence about such a relationship of LAD and LAD/BSA with the incidence of haemorrhagic stroke and its mortality, which has not been reported previously. The underlying mechanisms are not fully elucidated, but some hypotheses have been proposed, which might partially account for above associations. First, enlarged LA size may be a surrogate marker for cardiac and/or vascular weakening and damage, which are correlated with increased risk of stroke and mortality. ${ }^{11}{ }^{34}$ Second, LA dilation may promote blood stasis, which in turn predisposes to thrombus formation

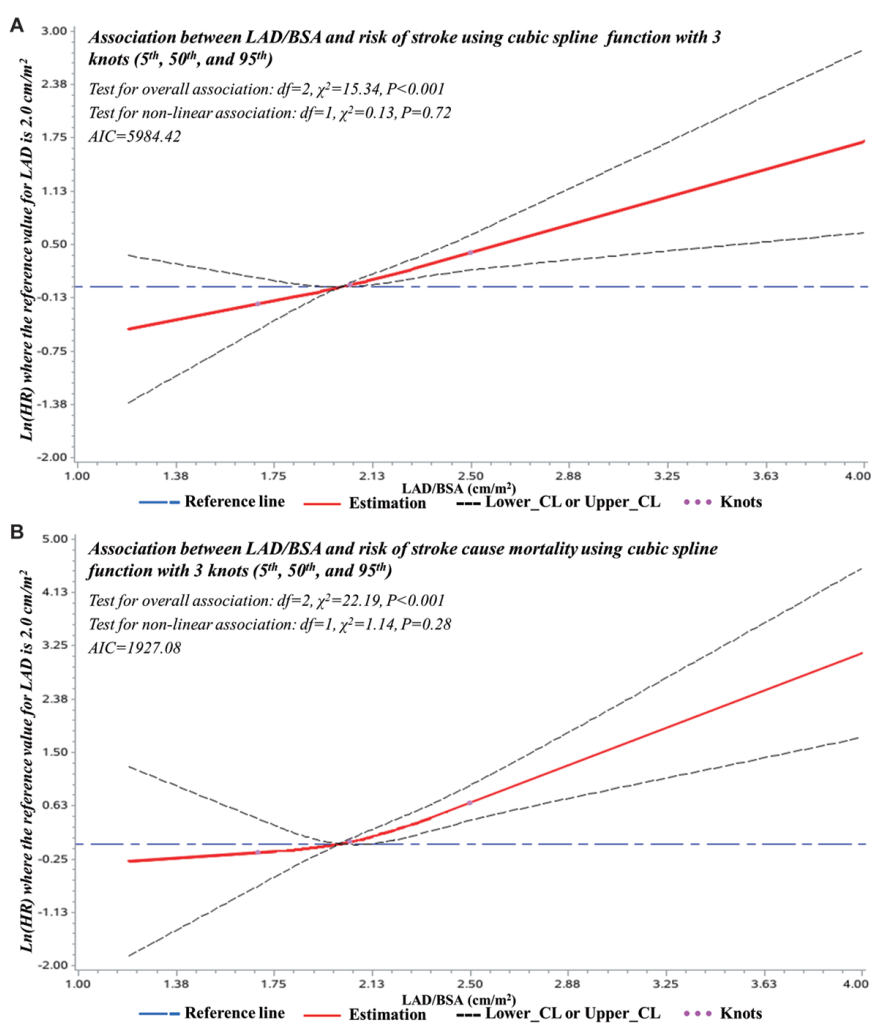

Figure 5 Adjusted dose-response associations between LAD/BSA and the risk of stroke (A) and stroke cause mortality (B). Adjusted for age, gender, BSA, smoking, drinking, heart rate, history of $\mathrm{CHD}$, history of $A F$, history of HF, history of heart valve diseases, hypertension, diabetes, medication for hypertension and diabetes, dyslipidaemia, eGFR, statin use, aspirin use, LVMI, LVEF, E/e' as appropriate. The Y-axis indicates the $\ln (\mathrm{HR})$ of stroke cause mortality for any value of LAD/BSA compared with the reference values. Dashed lines refer to the $95 \% \mathrm{Cls}$. AIC, Akaike information criterion; $\mathrm{CL}$, confidence interval; LAD, left atrial diameter; BSA, body surface area; $\mathrm{CHD}$, coronary heart disease; $\mathrm{AF}$, atrial fibrillation; HF, heart failure; eGFR, estimated glomerular filtration rate; LVMI, left ventricular mass index; LVEF, left ventricular ejection fraction.

and the potential for the embolisation, and carries its association with higher risk of ischaemic stroke. ${ }^{17} 3536$ Third, the most common cause of haemorrhagic stroke is hypertension, and LAE is often viewed as a consequence of exposure to hypertension burden. ${ }^{537}$ Additionally, oral anticoagulation therapy may contribute to the bleeding tendency in haemorrhagic stroke. ${ }^{38}{ }^{39}$ Our findings have possible clinical implications for stroke risk stratification, screening and stroke prevention.

Several limitations should be addressed. First, this was a single centre study and we enrolled subjects capable of receiving echocardiography, both of which perhaps caused selection bias. Second, although we adjusted for potential risk factors in the multivariate analysis, there might be residual confounders. Third, there was the lacking information on detailed stroke type (eg, cardioembolic) and stroke severity. Finally, the limited number of events due to short follow-up time might restrict the 
study conclusions. Then, larger multi-centre prospective researches with longer periods of follow-up are deserved to confirm our findings.

\section{CONCLUSION}

Our prospective cohort study found that subjects with LAE were more likely to display an elevated incidence of stroke and poor survival prognosis than those with normal $\mathrm{LAD}$. LAD and LAD/BSA were possible predictors for incident stroke and subsequent mortality in the general population and specific subgroups. Echocardiographic LA size, especially LAD and LAD/BSA, holds promise as a valuable means of predicting stroke and stroke cause mortality, and might be useful in developing diagnostic and preventive therapeutic strategies.

Acknowledgements We would like to thank Professor Liqiang Zheng for his help with data collection and data management.

Contributors TL participated in echocardiographic measurements and wrote the manuscript. GL conducted statistical analyses. XG assisted with critical revision of manuscript for intellectual content. ZL coordinated data collection and subjects' follow-up. JY contributed to echocardiographic analyses and readings. YS was responsible for the study concept and design. All authors have read and approved the final manuscript.

Funding This study was supported by grants from the National Key Research and Development Programme of China (2017YFC1307600), National Key Research and Development Programme from the Ministry of Science and Technology of China (2018YFC1312403), National Natural Science Foundation of China (82001828) and Natural Science Foundation of Liaoning Province (2020-BS-102).

Competing interests None declared.

Patient consent for publication Not required.

Ethics approval The study was approved by the Ethics Committee of China Medical University (Shenyang, China) (2018194). Written informed consent was obtained from all participants.

Provenance and peer review Not commissioned; externally peer reviewed. Data availability statement Data are available upon reasonable request.

Supplemental material This content has been supplied by the author(s). It has not been vetted by BMJ Publishing Group Limited (BMJ) and may not have been peer-reviewed. Any opinions or recommendations discussed are solely those of the author(s) and are not endorsed by BMJ. BMJ disclaims all liability and responsibility arising from any reliance placed on the content. Where the content includes any translated material, BMJ does not warrant the accuracy and reliability of the translations (including but not limited to local regulations, clinical guidelines, terminology, drug names and drug dosages), and is not responsible for any error and/or omissions arising from translation and adaptation or otherwise.

Open access This is an open access article distributed in accordance with the Creative Commons Attribution Non Commercial (CC BY-NC 4.0) license, which permits others to distribute, remix, adapt, build upon this work non-commercially, and license their derivative works on different terms, provided the original work is properly cited, appropriate credit is given, any changes made indicated, and the use is non-commercial. See: http://creativecommons.org/licenses/by-nc/4.0/.

ORCID iDs

Guangxiao Li http://orcid.org/0000-0003-0318-3490

Yingxian Sun http://orcid.org/0000-0002-2394-8625

\section{REFERENCES}

1 Feigin VL, Forouzanfar MH, Krishnamurthi R, et al. Global and regional burden of stroke during 1990-2010: findings from the global burden of disease study 2010. Lancet 2014;383:245-55.
2 Xu Y, Zhao L, Zhang L, et al. Left atrial enlargement and the risk of stroke: a meta-analysis of prospective cohort studies. Front Neurol 2020;11:26.

3 Khidhir AJ, Al-Shimmery EK, Alwan MH. Are left atrial abnormalities a risk for stroke? Neurosciences 2010;15:21-6.

4 Karas MG, Devereux RB, Wiebers DO, et al. Incremental value of biochemical and echocardiographic measures in prediction of ischemic stroke: the strong heart study. Stroke 2012;43:720-6.

$5 \mathrm{Kim} \mathrm{BS}$, Lee HJ, Kim JH, et al. Relationship between left atrial size and stroke in patients with sinus rhythm and preserved systolic function. Korean J Intern Med 2009;24:24-32.

6 Koh AS, Murthy VL, Sitek A, et al. Left atrial enlargement increases the risk of major adverse cardiac events independent of coronary vasodilator capacity. Eur J Nucl Med Mol Imaging 2015;42:1551-61.

7 Lang RM, Badano LP, Mor-Avi V, et al. Recommendations for cardiac chamber quantification by echocardiography in adults: an update from the American Society of echocardiography and the European association of cardiovascular imaging. J Am Soc Echocardiogr 2015;28:1-39.

8 Yaghi S, Moon YP, Mora-McLaughlin C, et al. Left atrial enlargement and stroke recurrence: the Northern Manhattan stroke study. Stroke 2015;46:1488-93.

9 Nagarajarao HS, Penman AD, Taylor HA, et al. The predictive value of left atrial size for incident ischemic stroke and all-cause mortality in African Americans: the Atherosclerosis risk in communities (ARIC) study. Stroke 2008;39:2701-6.

10 Gardin JM, McClelland R, Kitzman D, et al. M-mode echocardiographic predictors of six- to seven-year incidence of coronary heart disease, stroke, congestive heart failure, and mortality in an elderly cohort (the cardiovascular health study). Am J Cardiol 2001;87:1051-7.

11 Kizer JR, Bella JN, Palmieri V, et al. Left atrial diameter as an independent predictor of first clinical cardiovascular events in middle-aged and elderly adults: the strong heart study (SHS). Am Heart J 2006;151:412-8.

12 Kamel H, Bartz TM, Elkind MSV, et al. Atrial cardiopathy and the risk of ischemic stroke in the CHS (cardiovascular health study). Stroke 2018;49:980-6.

13 Olshansky B, Heller EN, Mitchell LB, et al. Are transthoracic echocardiographic parameters associated with atrial fibrillation recurrence or stroke? results from the atrial fibrillation follow-up investigation of rhythm management (affirm) study. J Am Coll Cardiol 2005;45:2026-33.

14 Benjamin EJ, D'Agostino RB, Belanger AJ, et al. Left atrial size and the risk of stroke and death. The Framingham heart study. Circulation 1995;92:835-41.

15 Di Tullio MR, Sacco RL, Sciacca RR, et al. Left atrial size and the risk of ischemic stroke in an ethnically mixed population. Stroke 1999;30:2019-24.

16 Bouzas-Mosquera A, Broullón FJ, Álvarez-García N, et al. Left atrial size and risk for all-cause mortality and ischemic stroke. CMAJ 2011;183:E657-64.

17 Hamatani Y, Ogawa H, Takabayashi K, et al. Left atrial enlargement is an independent predictor of stroke and systemic embolism in patients with non-valvular atrial fibrillation. Sci Rep 2016;6:31042.

18 Li Z, Guo X, Zheng L, et al. Grim status of hypertension in rural China: results from Northeast China rural cardiovascular health study 2013. J Am Soc Hypertens 2015;9:358-64.

19 Li Z, Guo X, Zheng L, et al. Prehypertension in rural northeastern China: results from the Northeast China rural cardiovascular health study. J Clin Hypertens 2014;16:664-70.

20 Chobanian AV, Bakris GL, Black HR, et al. The seventh report of the joint National Committee on prevention, detection, evaluation, and treatment of high blood pressure: the JNC 7 report. JAMA 2003;289:2560-72.

21 Diabetes Canada Clinical Practice Guidelines Expert Committee, Punthakee Z, Goldenberg R, et al. Definition, classification and diagnosis of diabetes, prediabetes and metabolic syndrome. Can $J$ Diabetes 2018;42:S10-15.

22 Expert Panel on Detection, Evaluation, and Treatment of High Blood Cholesterol in Adults. Executive summary of the third report of the National cholesterol education program (NCEP) expert panel on detection, evaluation, and treatment of high blood cholesterol in adults (adult treatment panel III). JAMA 2001;285:2486-97.

23 Yao G-H, Deng Y, Liu Y, et al. Echocardiographic measurements in normal Chinese adults focusing on cardiac chambers and great arteries: a prospective, nationwide, and multicenter study. J Am Soc Echocardiogr 2015;28:570-9.

24 Zhao D, Liu J, Wang W, et al. Epidemiological transition of stroke in China: twenty-one-year observational study from the Sino-MONICABeijing project. Stroke 2008;39:1668-74. 
25 Hoyo C, Cook MB, Kamangar F, et al. Body mass index in relation to oesophageal and oesophagogastric junction adenocarcinomas: a pooled analysis from the International BEACON Consortium. Int J Epidemiol 2012;41:1706-18.

26 Froehlich L, Meyre P, Aeschbacher S, et al. Left atrial dimension and cardiovascular outcomes in patients with and without atrial fibrillation: a systematic review and meta-analysis. Heart 2019;105:1884-91.

27 Polovina MM, Coats A, Seferovic P. Is left atrium the best kept secret of the heart? Left atrial dilatation and cardiovascular outcomes. Heart 2019;105:1848-9.

28 Chang A, Ricci B, Grory BM, et al. Cardiac biomarkers predict large vessel occlusion in patients with ischemic stroke. J Stroke Cerebrovasc Dis 2019;28:1726-31.

29 Goldstein LB. Left atrial enlargement: a cause of stroke? CMAJ 2011;183:1129-30.

30 Li G, Chi W, Bai B, et al. Dose-response associations between metabolic indexes and the risk of comorbid type 2 diabetes mellitus among rheumatoid arthritis patients from Northern China: a casecontrol study. BMJ Open 2019;9:e028011.

31 Mosquera VX, Bouzas-Mosquera A, González-Barbeito M, et al. Indexed left atrial size predicts all-cause and cardiovascular mortality in patients undergoing aortic valve surgery. J Thorac Cardiovasc Surg 2017;153:1275-84.
32 Yoshida K, Obokata M, Kurosawa K, et al. Effect of sex differences on the association between stroke risk and left atrial anatomy or mechanics in patients with atrial fibrillation. Circ Cardiovasc Imaging 2016;9.

33 Bushnell CD, Chaturvedi S, Gage KR, et al. Sex differences in stroke: challenges and opportunities. J Cereb Blood Flow Metab 2018;38:2179-91.

34 Pierdomenico SD, Pierdomenico AM, Di Carlo S, et al. Left atrial enlargement and risk of ischemic stroke in elderly treated hypertensive patients. Am J Hypertens 2014;27:1179-84.

35 Tufano A, Galderisi M. Can echocardiography improve the prediction of thromboembolic risk in atrial fibrillation? evidences and perspectives. Intern Emerg Med 2020;15:935-43.

36 Elkind MSV. Atrial cardiopathy and stroke prevention. Curr Cardiol Rep 2018;20:103.

37 Adeoye AM, Ogah OS, Ovbiagele B, et al. Prevalence and prognostic features of ECG abnormalities in acute stroke: findings from the siren study among Africans. Glob Heart 2017;12:99-105.

38 Chung MK, Refaat M, Shen W-K, et al. Atrial fibrillation: JACC Council perspectives. J Am Coll Cardiol 2020;75:1689-713.

39 Giede-Jeppe A, Roeder SS, Macha K, et al. Management of stroke in patients with left ventricular assist devices. J Stroke Cerebrovasc Dis 2020;29:105166. 